\title{
Negative Pressure Wound Therapy to the Dura
}

\author{
Ojas Pujji, Dr. Edmund Farrar, Prof. Steven Jeffery
}

Department of Burns and Plastic Surgery, University Hospital Birmingham, Birmingham, United Kingdom

\section{Introduction}

Negative-pressure wound therapy (NPWT) is historically contraindicated in patients with untreated osteomyelitis, necrotic tissue with eschar and precautioned in patients with weakened blood vessels. ${ }^{[1-2]}$ Although its beneficial effects on the scalp have been published, there is a lack of literature describing the application of NPWT on delicate vasculature and internal organ such as the dura mater. [3] Two cases which have shed light on this scenario by Khan et al. and Mohammed-Ali et al. where post-operative ischaemia following rupture of a right cerebral artery aneurysm and an extensive squamous cell carcinoma respectively were treated successfully with NPWT on the dura. ${ }^{[4][5]}$ However, application of NPWT on the dura with the mechanism of injury documented as a burn has not yet occurred.

\section{Case Report}

This was a 49-year-old gentleman with a background of multiple sclerosis, type 1 diabetes and cognitive impairment. 18 months previously the patient had fallen in front of a gas fire, and having been unable to get up due to his multiple sclerosis sustained full thickness burns to his right knee and scalp. He presented from his nursing home with a fever and infection of the scalp wound.
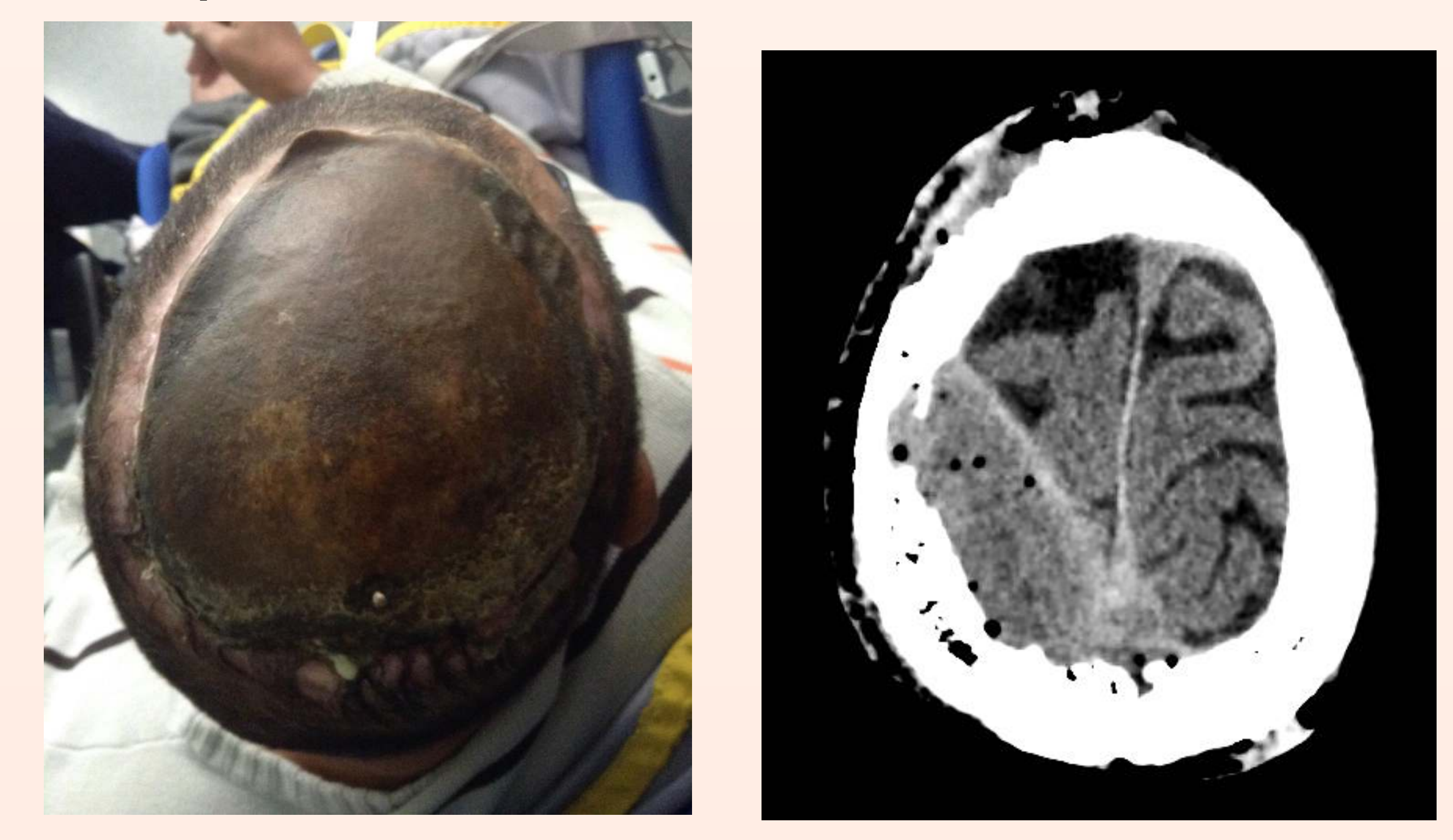

Upon admission, he was started on IV antibiotics. A CT head was undertaken to assess bony involvement, which showed a $1.2 \mathrm{~cm}$ high parietal extraaxial fluid/gas collection, deep to an abnormally lucent segment of parietal bone.
These in combination with the clinical presentation were highly suggestive of osteomyelitis and an infective collection.

\section{Operative Technique}

The central burn skin was excised; this was found to be necrotic. The exposed burn underneath showed small areas of pus discharging. A craniectomy of the central bone was performed, and again large amounts of pus discharged from the posterior incision. The bone was clearly eroded by infection. This was debrided to healthy bone.

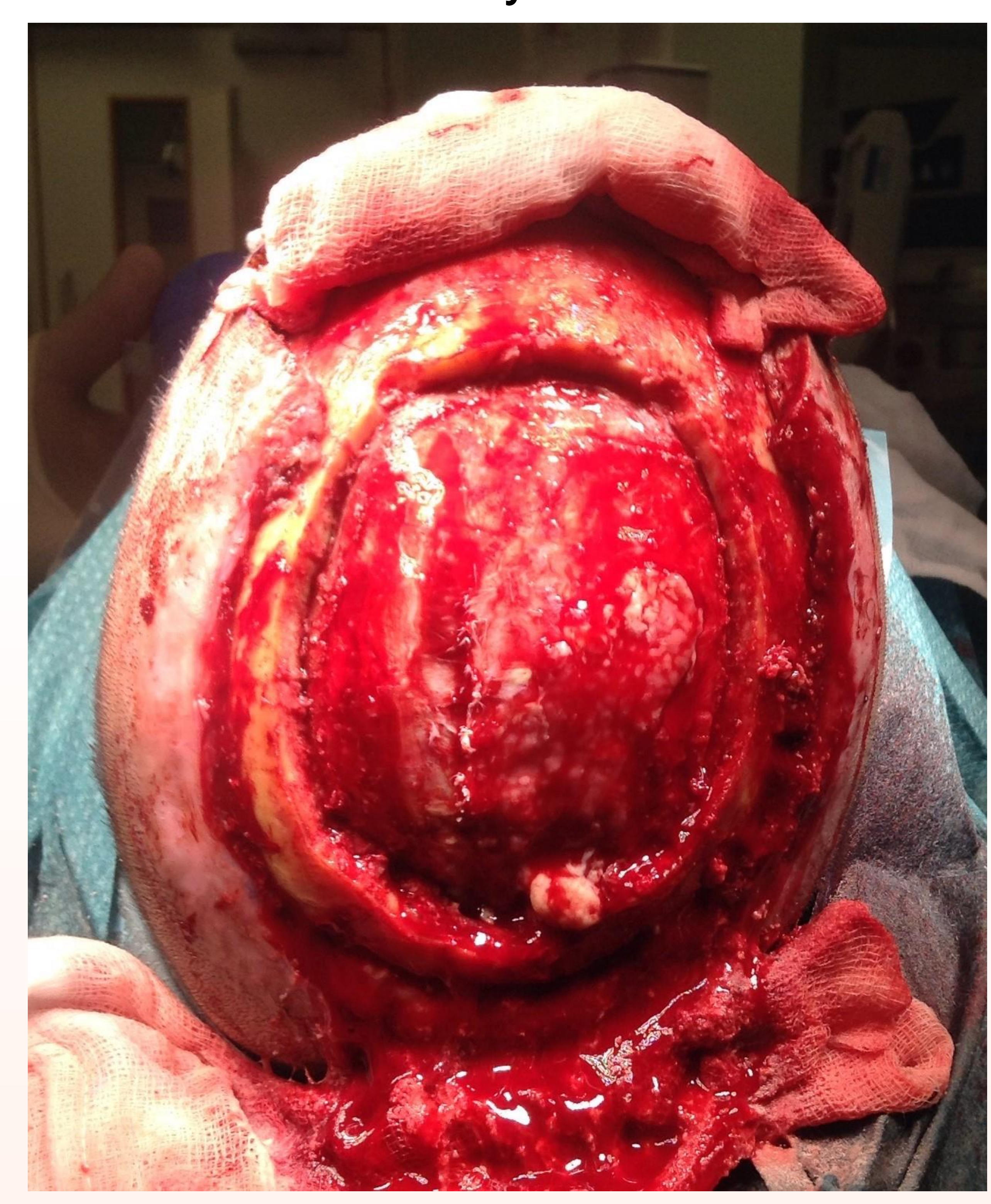

An NPWT dressing was then applied at $40 \mathrm{mmHg}$.

The dressing was changed weekly, until the three weeks point.

Integra was applied to the scalp and the NPWT dressing was replaced. The following week he returned for the second stage of the skin graft application.

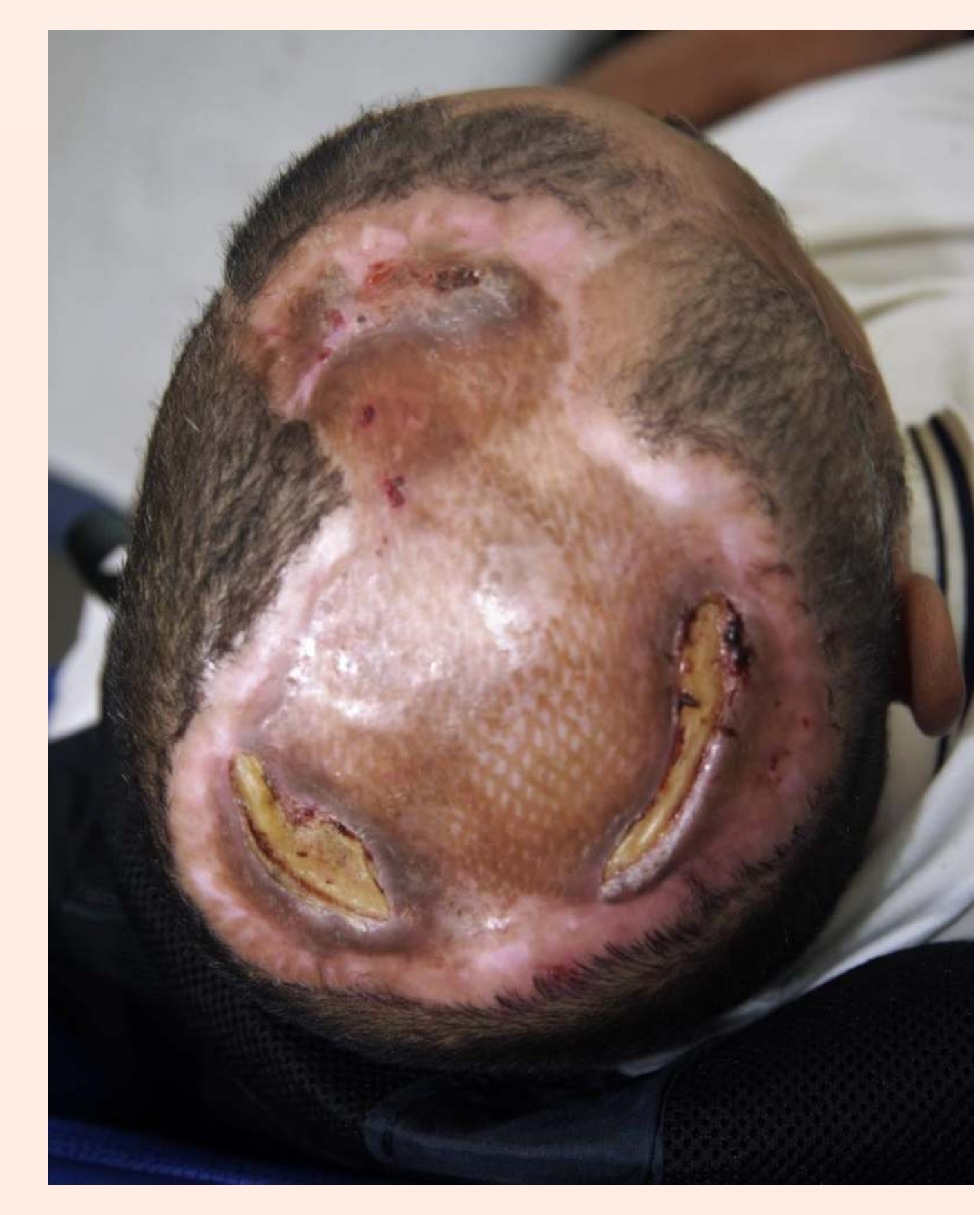

\section{Discussion}

The decision to use NPWT post-surgery was to prevent further infection in a high-risk patient, to isolate the wound and to capture the exudate. To ensure safe usage of NPWT all necrotic bone had to be debrided, the eschar removed and all exposed blood vessels were cauterised. The dura mater was kept intact: cases of hydrocephalus have been seen where NPWT was applied to a reapproximated dura. ${ }^{[6]}$

A further benefit of the NPWT was the reduction in the number of dressing changes required as it is applied for longer periods of time. This lessens the burden on the nursing staff which reduces costs and labour power required for dressing changes, this is especially apparent in burns ${ }^{[7]}$ and chronic wounds. ${ }^{[8]}$

The only significant postoperative complication in this case was a headache. This is a rare documented phenomenon in craniectomy due to sinking skin flap syndrome. The hospital's acute pain team were involved with the treatment. ${ }^{[9]}$ However, these symptoms did resolve and by the time discharge was planned pain was well under control.

\section{Conclusions}

The use of NPWT to the dura to aid wound healing in a deep burn of the scalp was shown to be beneficial in this patient.

\section{References}

[1] Henderson V, Timmons J, Hurd, T, Deroo K, Maloney S, Sabo S. NPWT in everyday practice Made Easy. Wounds International 2010; 1(5): Available from http://www.woundsinternational.com

[2] A N, Khan WS, J P. The Evidence-Based Principles of Negative Pressure Wound Therapy in Trauma \& Orthopedics. The Open Orthopaedics Journal. 2014;8:168-177. doi:10.2174/1874325001408010168.

[3] Mellott, A.J.; Zamierowski, D.S.; Andrews, B.T. Negative Pressure Wound Therapy in Maxillofacial Applications. Dent. J. 2016; 4: 30.

[4] Huang, C., Leavitt, T., Bayer, L.R., Orgill, D.P. Effect of negative pressure wound therapy on wound healing. Curr Probl Surg. 2014; 51(7), 301-331.

[5] John A. LoGiudice, MD, Jacob Thayer, BS, Alistair T. Hoyt, MD, Wade M. Mueller, MD; Use of a Dermal Regeneration Template in Complicated Craniotomy Wounds: Review of Series. Oper Neurosurg (Hagerstown). 2015; 11(4): 545-553.

[6] Powers AK, Neal MT, Argenta LC, Wilson JA, DeFranzo AJ, Tatter SB. Vacuum-assisted closure for complex cranial wounds involving the loss of dura mater. J Neurosurg. 2013; 118: 302-308.

[7] Jeffery SLA. Current burn wound management. Trauma. 2009; 11(4); 241-248. [8f Othman D. Negative Pressure Wound Therapy Literature Review of Efficacy, Cost Its Implementation in the United Kingdom. Plast Surg Int 2012; pp. 374-398. [9] Joseph V, Reilly P. Syndrome of the Trephined J Neurosurg, 2009; 111(4): 650-652 